\title{
The Future of Human-Food Interaction
}

\author{
Jialin Deng \\ Exertion Games Lab, Monash \\ University, Melbourne, Australia
}

\begin{abstract}
Ferran Altarriba Bertran
Social and Emotional Technology Lab, University of California, Santa Cruz, USA
\end{abstract}

\author{
Katherine Isbister \\ Department of Computational Media, \\ University of California, Santa Cruz,
} USA

\author{
Yan Wang \\ Exertion Games Lab, Monash \\ University, Melbourne, Australia
}

Rob Comber

KTH Royal Institute of Technology, Stockholm, Sweden

\author{
Charles Spence \\ Crossmodal Research Lab, \\ Department of Experimental \\ Psychology, Oxford University, \\ Oxford, UK
}

\author{
Carlos Velasco \\ Department of Marketing, BI \\ Norwegian Business School, Oslo, \\ Norway \\ Marianna Obrist \\ Department of Computer Science, \\ University College London, London, \\ UK
}

\author{
Florian 'Floyd' Mueller \\ Exertion Games Lab, Monash \\ University, Melbourne, Australia
}

\begin{abstract}
There is an increasing interest in food within the HCI discipline, with many interactive prototypes emerging that augment, extend and challenge the various ways people engage with food, ranging from growing plants, cooking ingredients, serving dishes and eating together. Grounding theory is also emerging that in particular draws from embodied interactions, highlighting the need to consider not only instrumental, but also experiential factors specific to human-food interactions. Considering this, we are provided with an opportunity to extend human-food interactions through knowledge gained from designing novel systems emerging through technical advances. This workshop aims to explore the possibility of bringing practitioners, researchers and theorists together to discuss the future of human-food interaction with a particular highlight on the design of experiential aspects of human-food interactions beyond the instrumental. This workshop extends prior community building efforts in this area and hence explicitly invites submissions concerning the empirically-informed knowledge of how technologies can enrich eating experiences. In doing so, people will benefit not only from new technologies around food, but also incorporate the many rich benefits that are associated with eating, especially when eating with others.
\end{abstract}

\section{CCS CONCEPTS}

- Human-centered computing; • Interaction design;

\section{KEYWORDS}

Food, eating, human-food interaction

\section{ACM Reference Format:}

Jialin Deng, Yan Wang, Carlos Velasco, Ferran Altarriba Bertran, Rob Comber, Marianna Obrist, Katherine Isbister, Charles Spence, and Florian 'Floyd' Mueller. 2021. The Future of Human-Food Interaction. In CHI Conference on Human Factors in Computing Systems Extended Abstracts (CHI '21 Extended Abstracts), May 08-13, 2021, Yokohama, Japan. ACM, New York, NY, USA, 6 pages. https://doi.org/10.1145/3411763.3441312

\section{BACKGROUND}

"One of the very nicest things about life is the way we must regularly stop whatever it is we are doing and devote our attention to eating." Luciano Pavarotti

Food is an essential part of life. From birth to death, we spend hours procuring, preparing, eating and digesting food [41]. Its preparation, consumption and even mere sight can bring immense joy $[9,30]$. French gastronome Brillat-Savarin argues that the pleasures associated with eating constitute some of life's most enjoyable experiences [8]. Recognizing this seems to be an upward trend, as most millennials identify themselves as "foodies" [42].

With advancements in technology, there has been an increase in the use of digital technology in regard to food-related practices ranging from how we grow, cook, present, eat and dispose of food [26]. Food practices are defined as any human activity in which food is involved, ranging from agriculture, food preparation, eating, gifting food, sharing meals and cleaning up. These are studied in HCI under the term of human-food interaction (FHI), defined as "the interconnection between the self and food" [12, 15].

Human-food interaction researchers have begun experimenting with emerging technologies like computational gastronomy [57], food printing [25, 46], virtual reality [4], capacitance sensing $[53,54]$, robotics [31], electrical muscle stimulation [34] acoustic levitation [51] and shape-changing interfaces [52] to illustrate new ways of interacting with food. These works highlight the increasing convergence of food and technology leading to new possibilities. At the same time, researchers in the fields of gastrophysics [45] and molecular gastronomy [3] have explored new ways of how restaurants can innovate in food design. These developments are supplemented by smart kitchens [33] and appliances [29, 46] that 
enable enhanced food preparation processes. At the same time, social media like Instagram and Facebook support the photographing of meals and sharing of cooking videos [11]. Practices of personalized nutrition [20] and DIY food sciences [28] are also gaining traction.

It is important to note that an HCI perspective on human-food interactions needs to consider the transdisciplinary nature of food research. For example, prior works from a range of fields have investigated human-food interactions, such as anthropology [23, 32], medical sciences $[24,44,56]$, psychology [5, 17, 37, 40], and sociology $[43,55]$. In consequence, we would like to create an interdisciplinary space for researchers to discuss human-food interaction, in an integral, comprehensive manner. We invite and welcome participants from such fields. We also recommend HCI researchers to consider references outside their immediate field.

Human-food interaction researchers have already collected prior work in workshops $[13,14,16,22,28,39]$, resulting in an online summary of existing research [7]. There are also emergent communities, for example, the FoodCHI Special Interest Group [26], the SIGCHI foodCHI network, and the ACM Future of Computing Academy working group on Computing and Food [38], and the Feeding Food Futures (FFF) network [2].

Several of the workshop organizers have been previously involved in conducting such workshops. For example, there was a workshop on "designing recipes for digital food cultures" [22] at CHI'18 that focused on recipes as part of human-food interactions. At CHI'17, there was a workshop called "digital health \& self-experimentation [21] that explored, amongst other topics, how to support health through food tracking. At the same conference, there was also a Special Interest Group (SIG) called "Future of Food in the Digital Realm" [26] that briefly brought human-food interaction researchers together at $\mathrm{CHI}$. Outside $\mathrm{CHI}$, related conferences and associated events have also experienced workshops and similar activities around the topic of food or eating, some of them run by organizers of this workshop. This includes workshops at ICMI [35, 36, 48-50], DIS [18, 19, 47], UbiComp'13 and '14 [14], CHI Play'19 [6], EFOOD'19 [1] and at IDC'20 [10].

These past activities highlight that there is an increasing interest in the merging of interactive technology and food, which has attracted attention in a variety of venues by a range of different people from different backgrounds. However, what has not yet taken place is a workshop at $\mathrm{CHI}$ that focuses exclusively on the topic of human-food interaction from a holistic perspective and hence considers not just individual aspects such as instrumental perspectives (e.g. health), but also welcomes submissions by people interested in the rich experiential perspectives and how to design for it, paying homage to the diverse community that $\mathrm{CHI}$ has been able to attract and bring together over the years. As such, this workshop takes a holistic approach to human-food interaction and highlights that there seems to be an underexplored area within prior workshops around the design of experiential aspects of human-food interactions and hence explicitly invites submissions concerning these.

To complement these prior approaches that were often limited by venue capacity, time and/or thematic focus, we are planning a full day of activities around human-food interaction at CHI'21. Given the increasing interest in human-food interaction, and the initiatives by different subgroups in the field, we believe it is time to join efforts and provide the foundations for this field of inquiry. With this workshop, we aim to bring together a diverse group of scholars to critically discuss past, present, and the future of humanfood interaction, as well as how technology design can contribute to food futures that are increasingly stimulating, sustainable, just, and socio-culturally rich.

\subsection{Topics of interest}

The topics of interest for the workshop include theories, technologies, and applications related to human-food interactions from an HCI perspective. We also encourage contributions that do not neatly fit in existing categories.

1.1.1 Theory, Methods and Aims. Theoretical topics of interest include, but are not limited to:

- Articulations of theoretical aspects of human-food interactions within existing HCI theories, such as embodied interactions

- Links to theories from non-HCI domains, such as health, multisensory perception, and food science

- The use of theoretical understandings to inform the design of human-food interactions, in particular experiential perspectives

- Critical reflections on the potential of, and risks derived from, integrating technology into our food lives and the food system at large

- Methods for co-designing food futures that are socially just, culturally and emotionally stimulating, and sustainable

1.1.2 Technology. We are also interested in contributions related to how technologies such as capacitance sensing, camera-based detection, etc. along with emerging techniques such as machine learning and artificial intelligence can be leveraged for sensing eating and associated activities around food. Such technology topics of interest include, but are not limited to:

- Sensing food

- Detecting eating

- Ingredient analysis

- Artificial intelligence and food

1.1.3 Applications of Food Technology. Applied topics of interest include, but are not limited to:

- Augmenting eating interactions

- Supplementing and enriching multisensory experiences

- Taste as feedback mechanism

- Food visualization

- Edibalization

- Food games and play

- Designing for restaurants, canteens and other food outlets

- Working with the hospitality industry

- Novel approaches and mechanisms for working with food

- Designing cyber food as part of human-machine integration 


\subsection{Goals of the Workshop}

The most important goal of the workshop is to provide an enduring community and networking platform for researchers who are interested in the coming together of food and interactive technology. Other workshop goals are to identify and articulate relevant theoretical insights and guide future research, understand synergies at the intersection of emerging technologies and current knowledge; nurture the growth of a cross-disciplinary research community around the topics and develop plans for subsequent activities (e.g. a journal special issue on the topic); and promote HFI design and research practices that are critical and sustainable from a social, cultural, and planetary perspective.

\section{ORGANIZERS}

2.1 Jialin Deng is a $\mathrm{PhD}$ candidate from the Exertion Games Lab in the Human-Centred Computing department at Monash University, Melbourne, Australia. Her research on interactive eating looks at the future of computational integrated food for an understanding of the design of a synergistic interplay between food and digital technology, as well as the impact of design and technology on the eating experience reflecting human subjectivity, culture, and identity. Jialin has a multidisciplinary background having worked at the intersection of art, design, and the food innovation industry. jialindeng.wixsite.com/whispery-savoury.

2.2 Yan Wang is a $\mathrm{PhD}$ candidate from the Exertion Games Lab in the Human-Centred Computing department at Monash University, Melbourne, Australia. Her research focuses on exploring how technologies might enrich eating experiences through augmented multisensory interactions and how playful designs could be developed to enrich eating experiences in everyday life. She has published extensively in the HFI field, demonstrated HFI inventions at $\mathrm{CHI}$ previously and has extensive workshop experience from SIGCHI conferences.

2.3 Carlos Velasco is an associate professor at the Department of Marketing, BI Norwegian Business School (Norway), where he co-founded the Centre for Multisensory Marketing. Carlos received his D.Phil. in Experimental Psychology from Oxford University. His work is at the intersection between Psychology, Marketing, and Human-Computer Interaction, and focuses on understanding, and capitalizing on, our multisensory experiences and their guiding principles.Carlos has worked with a number of companies from around the world on multisensory experiences. carlosvelasco.info/.

2.4 Ferran Altarriba Bertran is a $\mathrm{PhD}$ candidate in the Social and Emotional Technology Lab at UC Santa Cruz, USA. His research explores how future technologies might support increasingly playful relationships between people, and how situated codesign methods could be leveraged to develop them. As part of his research, Ferran speculates increasingly playful human-food interaction futures, looking at how technology can enable novel exciting forms of playful engagement with, through and around food that bring about positive social, cultural, and emotional outcomes. Ferran co-initiated the Feeding Food Futures network and co-organized several HFI workshops at DIS, CHI Play, IDC and EFOOD. ferranaltarriba.com.
2.5 Rob Comber is Associate Professor of Communication at KTH Royal Institute of Technology, Stockholm, Sweden. His research lies at the intersections of food, democracy and sustainability. His work is increasingly concerned with the interconnected nature of our food practices and the social and societal consequences of how we design for it. He was a founding member of the HumanFood Interaction community in SIGCHI, and has organised workshops on food in HCI at CHI'12, '13, '14, '15 and '16, DIS'12 and UbiComp'13 and '14. He was guest editor of the 2014 Special Issue on Designing for Human-Food Interaction in IJHCS.

2.6 Marianna Obrist is Professor of Multisensory Interfaces at UCL, Department of Computer Science, and Deputy Director (Digital Health) for the UCL Institute of Healthcare Engineering. She is investigating touch, taste, and smell as interaction modalities for HCI. She is a co-founder of OWidgets Ltd, a University start-up developing novel software and hardware solutions for smell experience design. She is an inaugural member of the ACM Future of Computing Academy and was selected Young Scientist 2017 and 2018 to attend the World Economic Forum. She is a Visiting Professor at the Royal College of Art and has recently published a book on 'Multisensory Experiences: where the senses meet technology'. multi-sensory.info.

2.7 Katherine Isbister is a Human-Computer Interaction and games researcher who creates and studies digital games and other playful computer-supported experiences. She is currently a full professor in the Department of Computational Media at the University of California, Santa Cruz, where she directs the Center for Computational Experience. Her focus is emotion and social connection-understanding the impact of design choices on these qualities, and getting better at building and evaluating technology that supports and enhances social and emotional experience.

2.8 Charles Spence is a world-famous experimental psychologist with a specialization in neuroscience-inspired multisensory design. He has worked with many of the world's largest companies across the globe since establishing the Crossmodal Research Laboratory (CRL) at the Department of Experimental Psychology, Oxford University in 1997. Prof. Spence has published over 1,000 academic articles and edited or authored 15 books including, in 2014, the Prose prize-winning "The perfect meal", and the international bestseller "Gastrophysics: The new science of eating" (2017; Penguin Viking) - winner of the 2019 Le Grand Prix de la Culture Gastronomique from Académie Internationale de la Gastronomie. He works in the interaction between food and technology, and has organized many workshops in this space, including being a co-organizer for the last four ICMI Food-Technology symposia.

2.9 Florian 'Floyd' Mueller is Professor in the Human-Centred Computing department at Monash University, Melbourne, Australia, where he directs the Exertion Games Lab. His research sits on the intersection between the human body, technology and play, aiming to help people live a fulfilling life, which includes savoring food. He has co-authored a "Foundation and Trends" treatise on human-food interaction [27] and researches how to experience eating as play. $\mathrm{He}$ has co-organized nine workshops at $\mathrm{CHI}$ previously and was general co-chair CHI'20. 


\section{WEBSITE}

The workshop website is at: humanfoodinteraction.org

The workshop website contains more details and the call for papers. It also promotes the workshop and presents relevant research results to date. All accepted contributions will appear on the website. Following the workshop itself, videos, photos, and results from the workshop activities will be added, with participants' consent.

\section{PRE-WORKSHOP PLANS}

We will publish a call for participation on the workshop website and circulate it widely to individuals and communities interested in the topic of the workshop. It will be open to both academic researchers as well as industry professionals and independent researchers. The call for participation will also be posted to mailing lists (including chi-announcements), social media and directly emailed to researchers in our networks. Participants will be expected to have done small tasks based on our brief before the workshop and to arrive with relevant questions and discussion points.

\section{WORKSHOP STRUCTURE}

CHI 2021 will be an entirely virtual conference because of the ongoing COVID-19 pandemic. It has given us an opportunity to run a very different kind of workshop. We have modified the structure and flow of the workshop to suit synchronous and asynchronous participation for attendees from multiple time zones, and it will be taking place virtually via Zoom or similar platforms. We will send through an email invitation with guidelines of attendance prior to the workshop. The duration of this online workshop will be approximately 4 hours in total. Activities in each session include:

1) Themed presentations: The themed presentations involve creating a shared understanding around the related theme of Human-Food Interaction in HCI and amongst the workshop group. The themes will draw from the growing research in Human-Food Interaction, which have focused on rather independent areas: Sensing Food, Design with Food, and Design around Food. The themed presentations will be given by at least one organizer (as theme facilitator) for each theme respectively, followed by group activities by participants.

2) Group interactions and exercises: The group-based interactions and exercises will be carried out in 3 small groups of around 6 people, each group will be assigned 1-2 organizers as facilitators. The group will brainstorm on at least one novel scenario or idea that focuses on novel human-food interactions, particularly considering experiential perspectives under the particular theme assigned to the group.

3) Showcasing and discussion: The deliverables will be a short write-up with drawings or illustrations to show how future technology could assist in the contexts and activities in question. Lastly, the concepts will be showcased and shared by each group, and then discussed. The organizers will provide working materials (whiteboards and working sheets) via online collaboration tools (e.g. Miro, Mura, and Google Docs). The whole process of this online workshop will be recorded and posted online with each participant's consent.

\subsection{Tentative Workshop Agenda:}

$\begin{array}{ll}\text { 10min: } & \text { Opening } \\ \text { 15min: } & \text { Introduction and ice-breaking activities }\end{array}$

Session 1

Theme 1: Sensing Food

10min: Presentation (one guest speaker)

20min: Group activities (ideation)

10min: Summary\&discussion (Q\&A)

Theme 2: Design with Food

10min: Presentation (one guest speaker)

20min: Group activities (ideation)

10min: $\quad$ Summary\&discussion (Q\&A)

Theme 3: Design around Food

10min: Presentation (one guest speaker)

20min: Group activities (ideation)

10min: Summary\&discussion (Q\&A)

10min: Virtual coffee break

Session 2

60min: Group activities (action)

10min: Virtual coffee break

30min: Showcasing and discussion

20min: Summary and closing

Total: $\quad 5 \mathrm{~h} 10 \mathrm{~min}$

\section{POST-WORKSHOP PLANS}

Workshop papers will be listed on the workshop website. A report on workshop activities, as well as selected photos and descriptions of outputs from the group activities (subject to authors' permissions) will also be published on the workshop website. We also aim to include social media platforms for continuing knowledge sharing based on the preferences of the community. A variety of post-workshop academic publications will be considered based on the content of the accepted submissions and the outputs of workshop sessions. These include, but are not limited to, a report to be submitted to a venue that is relevant to the $\mathrm{CHI}$ community (e.g. ACM Interactions), a full paper submission to a relevant SIGCHI conference based on synergies between individual efforts presented at the workshop, and a special issue of a journal informed by the research agendas articulated at the workshop.

\section{CALL FOR PARTICIPATION}

Human-Food Interaction, that is, the merging of interactive technology with food practices is becoming increasingly prevalent and popular, fueled by technical advances that make it possible to sense what and how people eat. Knowledge from a wealth of disciplines is required to design for these experiences, and communication between these disciplines is essential to positively inform future human-food interactions. To this end, the interdisciplinary workshop "The Future of Human-Food Interaction" aims to bring together diverse opinions and expertise to offer a platform for researchers and practitioners to learn from each other, highlighting the design of experiential perspectives of engaging with food. We welcome diverse contributions, including empirical research, engineering investigations, design concepts, theory, opinions, and reviews. For more information, please see: humanfoodinteraction.org 


\section{REFERENCES}

[1] Chasing Playful Inspiration In Culture And Traditions To Inspire Food Design. 2019, from: http://efood.fa.ulisboa.pt/index.php/14-workshop.

[2] Feeding Food Futures from: https://foodfutures.group/.

[3] Adrià, Ferran, Juli Soler, and Albert Adrià, El Bulli 2005-2011. 2014 Phaidon.

[4] Arnold, Peter, Rohit Ashok Khot, and Florian Mueller. 2018. "You Better Eat to Survive": Exploring Cooperative Eating in Virtual Reality Games. In Proceedings of the Twelfth International Conference on Tangible, Embedded, and Embodied Interaction, Stockholm, Sweden. Association for Computing Machinery, New York, NY, USA. 398-408. DOI: https://dx.doi.org/10.1145/3173225.3173238.

[5] Bays, Jan Chozen, Mindful Eating: A Guide to Rediscovering a Healthy and Joyful Relationship with Food (Revised Edition). 2017 Shambhala Publications.

[6] Bertran, Ferran Altarriba, Jared Duval, Katherine Isbister, Danielle Wilde, Elena Márquez Segura, Oscar Garcia Pañella, and Laia Badal León. 2019, Chasing Play Potentials in Food Culture to Inspire Technology Design. In Extended Abstracts of the Annual Symposium on Computer-Human Interaction in Play Companion Extended Abstracts (CHI PLAY '19 Extended Abstracts), Barcelona, Spain, Association for Computing Machinery, New York, NY, USA. 829-834. DOI: https://dx.doi.org/10.1145/3341215.3349586.

[7] Bertran, Ferran Altarriba, Samvid Jhaveri, Rosa Lutz, Katherine Isbister, and Danielle Wilde. 2019. Making Sense of Human-Food Interaction. In Proceedings of the 2019 CHI Conference on Human Factors in Computing Systems, Glasgow, Scotland Uk. Association for Computing Machinery, New York, NY, USA. Paper 678, 1-13. DOI: https://dx.doi.org/10.1145/3290605.3300908.

[8] Brillat-Savarin, Jean Anthelme, The physiology of taste: or meditations on transcendental gastronomy. 2009 Vintage.

[9] Cabanac, Michel, The dialectics of pleasure. Pleasures of the brain. 2010. 113-124.

[10] Chisik, Yoram, Ferran Altarriba Bertran, Marie-Monique Schaper, Elena Márquez Segura, Laia Turmo Vidal, and Danielle Wilde. 2020, Chasing play potentials in food culture: embracing children's perspectives. In Proceedings of the $2020 \mathrm{ACM}$ Interaction Design and Children Conference: Extended Abstracts (IDC '20), London, United Kingdom, Association for Computing Machinery, New York, NY, USA 46-53. DOI: https://dx.doi.org/10.1145/3397617.3398062.

[11] Choe, Hanwool, 2019, Eating together multimodally: Collaborative eating in mukbang, a Korean livestream of eating. Language in Society, 48(2): pp. 171-208. https://dx.doi.org/10.1017/S0047404518001355.

[12] Choi, Jaz Hee-jeong, Marcus Foth, and Greg Hearn, Eat, cook, grow: Mixing human-computer interactions with human-food interactions. 2014 MIT Press.

[13] Choi, Jaz Hee-jeong, Conor Linehan, Rob Comber, and John McCarthy. 2012. Food for thought: designing for critical reflection on food practices. In Proceedings of the Designing Interactive Systems Conference, Newcastle Upon Tyne, United Kingdom. Association for Computing Machinery, New York, NY, USA. 793-794. DOI: https://dx.doi.org/10.1145/2317956.2318077.

[14] Clear, Adrian K., Rob Comber, Adrian Friday, Eva Ganglbauer, Mike Hazas, and Yvonne Rogers. 2013. Green food technology: UbiComp opportunities for reducing the environmental impacts of food. In Proceedings of the 2013 ACM conference on Pervasive and ubiquitous computing adjunct publication, Zurich, Switzerland. Association for Computing Machinery, New York, NY, USA. 553-558. DOI https://dx.doi.org/10.1145/2494091.2497316.

[15] Comber, Rob, Jaz Hee-jeong Choi, Jettie Hoonhout, and Kenton O'Hara, 2014 Designing for human-food interaction: An introduction to the special issue on 'food and interaction design'. International fournal of Human-Computer Studies, 72(2): pp. 181-184. https://doi.org/10.1016/j.ijhcs.2013.09.001.

[16] Comber, Rob, Eva Ganglbauer, Jaz Hee-jeong Choi, Jettie Hoonhout, Yvonne Rogers, Kenton O'Hara, and Julie Maitland. 2012, Food and interaction design designing for food in everyday life. In CHI '12 Extended Abstracts on Human Factors in Computing Systems, Austin, Texas, USA, Association for Computing Machinery, New York, NY, USA. 2767-2770. DOI: https://dx.doi.org/10.1145/2212776.2212716.

[17] Connor, Mark and Christopher J Armitage, The Social Psychology of Food. 2002 Open University Press.

[18] Davis, Hilary, Danielle Wilde, Ferran Altarriba Bertran, and Markéta Dolejšová. 2020, Fantastic(e)ating Food Futures: Reimagining Human Food Interaction. In Companion Publication of the 2020 ACM Designing Interactive Systems Conference (DIS' 20 Companion), Eindhoven, Netherlands, Association for Computing Machinery, New York, NY, USA. 377-380. DOI: https://dx.doi.org/10.1145/3393914. 3395906.

[19] Dolejšová, Markéta, Ferran Altarriba Bertran, Danielle Wilde, and Hilary Davis. 2019, Crafting and Tasting Issues in Everyday Human-Food Interactions. In Companion Publication of the 2019 on Designing Interactive Systems Conference 2019 Companion (DIS '19 Companion), San Diego, CA, USA, Association for Computing Machinery, New York, NY, USA. 361-364. DOI: https://dx.doi.org/10. 1145/3301019.3319994.

[20] Dolejšová, Markéta and Denisa Kera. 2017. Soylent Diet Self-Experimentation: Design Challenges in Extreme Citizen Science Projects. In Proceedings of the 2017 ACM Conference on Computer Supported Cooperative Work and Social Computing, Portland, Oregon, USA. Association for Computing Machinery, New York, NY, USA. 2112-2123. DOI: https://dx.doi.org/10.1145/2998181.2998365.
[21] Dolejsova, Marketa, Denisa Kera, Cristiano Storni, Rohit Ashok Khot, Ivan John Clement, Inka Pavelka, and Puneet Kishor. 2017. Digital Health \& Selfexperimentation: Design Challenges \& Provocations. In Proceedings of the 2017 CHI Conference Extended Abstracts on Human Factors in Computing Systems (CHI EA '17), Denver, Colorado, USA. Association for Computing Machinery, New York, NY, USA. 510-517. DOI: https://dx.doi.org/10.1145/3027063.3027071.

[22] Dolejšová, Markéta, Rohit Ashok Khot, Hilary Davis, Hasan Shahid Ferdous, and Andrew Quitmeyer. 2018, Designing Recipes for Digital Food Futures. In Extended Abstracts of the 2018 CHI Conference on Human Factors in Computing Systems (CHI EA '18), Montreal QC, Canada, Association for Computing Machinery, New York, NY, USA. Paper W10. DOI: https://dx.doi.org/10.1145/3170427.3170622.

[23] Holtzman, Jon D., 2006, Food and Memory. Annual Review of Anthropology, 35(1): pp. 361-378. https://dx.doi.org/10.1146/annurev.anthro.35.081705.123220.

[24] Kendrick, Malcolm, The great cholesterol con: the truth about what really causes heart disease and how to avoid it. 2008 Kings Road Publishing.

[25] Khot, Rohit Ashok, Deepti Aggarwal, Ryan Pennings, Larissa Hjorth, and Florian Mueller. 2017. Edipulse: investigating a playful approach to self-monitoring through 3D printed chocolate treats. In Proceedings of the 2017 CHI Conference on Human Factors in Computing Systems, 6593-6607. DOI: https://doi.org/10.1145/ 3025453.3025980

[26] Khot, Rohit Ashok, Deborah Lupton, Markéta Dolejšová, and Florian Mueller. 2017. Future of Food in the Digital Realm. In Proceedings of the 2017 CHI Conference Extended Abstracts on Human Factors in Computing Systems, Denver, Colorado, USA. Association for Computing Machinery, New York, NY, USA. 1342-1345. DOI: https://dx.doi.org/10.1145/3027063.3049283.

[27] Khot, Rohit, Florian Mueller, and Damon Young, 2019, Human-Food Interaction. Foundations and Trends®in Human-Computer Interaction, 12: pp. 238-415. https: //dx.doi.org/10.1561/1100000074.

[28] Kuznetsov, Stacey, Christina J. Santana, Elenore Long, Rob Comber, and Carl DiSalvo. 2016. The Art of Everyday Food Science: Foraging for Design Opportunities. In Proceedings of the 2016 CHI Conference Extended Abstracts on Human Factors in Computing Systems, San fose, California, USA. Association for Computing Machinery, New York, NY, USA. 3516-3523. DOI: https://dx.doi.org/10.1145/ 2851581.2856483

[29] Laursen, Christian Østergaard, Søren Pedersen, Timothy Merritt, and Ole Caprani, 2016, Springer International Publishing, Cham, Robot-Supported Food Experiences. International Workshop in Cultural Robotics, vol 9549 pp. 107-130. https: //dx.doi.org/10.1007/978-3-319-42945-8_10.

[30] Lupton, Deborah, Food, The Body and the Self. Vol. 14. 1996.

[31] Mehta, Yash Dhanpal, Rohit Ashok Khot, Rakesh Patibanda, and Florian Mueller. 2018. Arm-A-Dine: Towards Understanding the Design of Playful Embodied Eating Experiences. In Proceedings of the 2018 Annual Symposium on ComputerHuman Interaction in Play, Melbourne, VIC, Australia. Association for Computing Machinery, New York, NY, USA. 299-313. DOI: https://dx.doi.org/10.1145/3242671. 3242710 .

[32] Mintz, Sidney W. and Christine M. Du Bois, 2002, The Anthropology of Food and Eating. Annual Review of Anthropology, 31(1): pp. 99-119. https://dx.doi.org/10. 1146/annurev.anthro.32.032702.131011.

[33] Mizrahi, Moran, Amos Golan, Ariel Bezaleli Mizrahi, Rotem Gruber, Alexander Zoonder Lachnise, and Amit Zoran. 2016, Digital Gastronomy: Methods \& Recipes for Hybrid Cooking. In In Proceedings of the 29th Annual Symposium on User Interface Software and Technology (UIST '16), Tokyo, Japan, Association for Computing Machinery, New York, NY, USA. 541-552. DOI: https://dx.doi.org/10.1145/2984511.2984528.

[34] Niijima, A. and T. Ogawa. 2016, A proposal of virtual food texture by electric muscle stimulation. In 2016 IEEE International Conference on Multimedia \& Expo Workshops (ICMEW), Seattle, WA, 1-6. DOI: https://dx.doi.org/10.1109/ICMEW. 2016.7574698.

[35] Nijholt, A., C. Velasco, G. Huisman, and K. Karunanayaka (Eds.). 2016, Proceedings of the 1st Workshop on Multi-sensorial Approaches to Human-Food Interaction. In Association for Computing Machinery, New York, NY, USA.

[36] Nijholt, Anton, Carlos Velasco, Marianna Obrist, Katsunori Okajima, and Charles Spence. 2018, 3rd International Workshop on Multisensory Approaches to Human-Food Interaction. In Proceedings of the 20th ACM International Conference on Multimodal Interaction (ICMI '18), Boulder, CO, USA, Association for Computing Machinery, New York, NY, USA,. 657-659. DOI: https://dx.doi.org/10.1145/ 3242969.3265860

[37] Obrist, Marianna, Rob Comber, Sriram Subramanian, Betina Piqueras-Fiszman, Carlos Velasco, and Charles Spence. 2014, Temporal, affective, and embodied characteristics of taste experiences: a framework for design. In Proceedings of the SIGCHI Conference on Human Factors in Computing Systems (CHI '14), Toronto, Ontario, Canada, Association for Computing Machinery, New York, NY, USA. 2853-2862. DOI: https://dx.doi.org/10.1145/2556288.2557007.

[38] Obrist, Marianna, Patrizia Marti, Carlos Velasco, Yunwen Tu, Takuji Narumi, and Naja L. Holten Møller. 2018, The future of computing and food: extended abstract. In Proceedings of the 2018 International Conference on Advanced Visual Interfaces (AVI '18), Castiglione della Pescaia, Grosseto, Italy, Association for Computing Machinery, New York, NY, USA. Article 5, 1-3. DOI: https://dx.doi.org/10.1145/ 3206505.3206605 . 
[39] Raturi, Ankita, Juliet Norton, Bill Tomlinson, Eli Blevis, and Lynn Dombrowski. 2017. Designing Sustainable Food Systems. In Proceedings of the 2017 CHI Conference Extended Abstracts on Human Factors in Computing Systems (CHI EA '17), Denver, Colorado, USA. Association for Computing Machinery, New York, NY, USA. 609-616. DOI: https://dx.doi.org/10.1145/3027063.3027075.

[40] Rogers, P. J., D. Ferriday, S. A. Jebb, and J. M. Brunstrom, 2016, Connecting biology with psychology to make sense of appetite control. Nutrition Bulletin, 41(4): pp 344-352. https://dx.doi.org/10.1111/nbu.12237.

[41] Rozin, Paul, Rebecca Bauer, and Dana Catanese, 2003, Food and life, pleasure and worry, among American college students: Gender differences and regional similarities. Fournal of Personality and Social Psychology, 85(1): pp. 132-141. https: //dx.doi.org/10.1037/0022-3514.85.1.132.

[42] Sarasohn-Kahn, J, We Are All Foodies Now. Retrieved March. Vol. 24. 2016. 2017.

[43] Schneider, Tanja, Food and Health, In: The Blackwell Encyclopedia of Sociology. (2nd Edition). 2018

[44] Scrinis, Gyorgy, Nutritionism: The science and politics of dietary advice. 2013 Columbia University Press.

[45] Spence, Charles, Gastrophysics: The new science of eating. 2017 Penguin UK.

[46] Sun, Jie, Zhuo Peng, Weibiao Zhou, Jerry Y. H. Fuh, Geok Soon Hong, and Annette Chiu, 2015, A Review on 3D Printing for Customized Food Fabrication. Procedia Manufacturing, 1: pp. 308-319. https://doi.org/10.1016/j.promfg.2015.09.057.

[47] Vannucci, Erica, Ferran Altarriba, Justin Marshall, and Danielle Wilde. 2018, Handmaking Food Ideals: Crafting the Design of Future Food-related Technologies. In Proceedings of the 2018 ACM Conference Companion Publication on Designing Interactive Systems (DIS '18 Companion), Hong Kong, China, Association for Computing Machinery, New York, USA. 419-422. DOI: https: //dx.doi.org/10.1145/3197391.3197403.

[48] Velasco, Carlos, Felipe Reinoso Carvalho, Olivia Petit, and Anton Nijholt. 2016, A multisensory approach for the design of food and drink enhancing sonic systems. In Proceedings of the 1st Workshop on Multi-sensorial Approaches to Human-Food Interaction (MHFI '16), Tokyo, Japan, Association for Computing Machinery, New York, NY, USA. Article 7. DOI: https://dx.doi.org/10.1145/3007577.3007578.

[49] Velasco, Carlos, Kasun Karunanayaka, and Anton Nijholt, 2018, Editorial: Multisensory Human-Food Interaction. Frontiers in Psychology, 9. https://dx.doi.org/ 10.3389/fpsyg.2018.00796.
[50] Velasco, Carlos, Anton Nijholt, Marianna Obrist, Katsunori Okajima, Rick Schifferstein, and Charles Spence. 2017, MHFI 2017: 2nd international workshop on multisensorial approaches to human-food interaction (workshop summary). In Proceedings of the 19th ACM International Conference on Multimodal Interaction (ICMI '17), Glasgow, UK, Association for Computing Machinery, New York, NY, USA. 674-676. DOI: https://dx.doi.org/10.1145/3136755.3137023.

[51] Vi, Chi Thanh, Asier Marzo, Damien Ablart, Gianluca Memoli, Sriram Subramanian, Bruce Drinkwater, and Marianna Obrist. 2017. TastyFloats: A Contactless Food Delivery System. In Proceedings of the 2017 ACM International Conference on Interactive Surfaces and Spaces, Brighton, United Kingdom. Association for Computing Machinery, New York, NY, USA. 161-170. DOI: https: //dx.doi.org/10.1145/3132272.3134123.

[52] Wang, Wen, Lining Yao, Teng Zhang, Chin-Yi Cheng, Daniel Levine, and Hiroshi Ishii. 2017. Transformative appetite: shape-changing food transforms from 2D to 3D by water interaction through cooking. In Proceedings of the the $2017 \mathrm{CHI}$ Conference on Human Factors in Computing Systems, 6123-6132. DOI: http://dx. doi.org/10.1145/3025453.3026019.

[53] Wang, Yan, Zhuying Li, Robert Jarvis, Rohit Ashok Khot, and Florian Mueller. 2018, The Singing Carrot: Designing Playful Experiences with Food Sounds. In Proceedings of the 2018 Annual Symposium on Computer-Human Interaction in Play Companion Extended Abstracts (CHI PLAY'18 Extended Abstracts), Melbourne, VIC, Australia, Association for Computing Machinery, New York, NY, USA. 669-676. DOI: https://dx.doi.org/10.1145/3270316.3271512.

[54] Wang, Yan, Zhuying Li, Robert S. Jarvis, Joseph La Delfa, Rohit Ashok Khot, and Florian Mueller. 2020, WeScream! Toward Understanding the Design of Playful Social Gustosonic Experiences with Ice Cream (DIS '20). In Proceedings of the 2020 ACM Designing Interactive Systems Conference, Eindhoven, Netherlands, Association for Computing Machinery, New York, NY, USA. 951-963. DOI: https: //dx.doi.org/10.1145/3357236.3395456.

[55] Warde, Alan, The Practice of Eating. 2016 John Wiley \& Sons.

[56] Willett, Walter C. and Meir J. Stampfer, 2013, Current Evidence on Healthy Eating. Annual Review of Public Health, 34(1): pp. 77-95. https://dx.doi.org/10. 1146/annurev-publhealth-031811-124646.

[57] Zoran, A., 2019, Cooking With Computers: The Vision of Digital Gastronomy [Point of View]. Proceedings of the IEEE, 107(8): pp. 1467-1473. https://dx.doi.org/ 10.1109/JPROC.2019.2925262. 